\title{
Clinical criteria for three types of dementia had low sensitivity and high specificity
}

\author{
Holmes C, Cairns N, Lantos P, et al. Validity of current clinical criteria for Alzheimer's disease, vascular dementia and dementia \\ with Lewy bodies. Br J Psychiatry 1999 Jan;174:45-50.
}

\section{Questions}

How valid are the following diagnostic criteria: (1) the NINCDSADRDA criteria for Alzheimer's disease (AD); (2) the NINDSAIREN criteria for vascular dementia $(\mathrm{VaD})$; and (3) the consensus criteria for dementia with Lewy bodies (CDLB) for dementia with Lewy bodies (DLB)? Do prevalence rates and copathology compromise these criteria?

\section{Design}

Blinded comparison of the resulting diagnoses using the NINCDS-ADRDA criteria, the NINDS-AIREN criteria, and the CDLB with the diagnostic standard, postmortem examination.

\section{Setting}

Community based in the UK.

\section{Patients}

The first 80 patients (58\% women) from the Camberwell Dementia Case Register to come to postmortem (mean age at death 82 y).

\section{Description of tests and diagnostic standard}

Clinical diagnosis was made independently by 2 psychiatrists, blind to postmortem findings. Diagnostic criteria of the
NINCDS-ADRDA for AD, the NINDS-AIREN for VaD, and the CDLB for DLB were used.

\section{Main outcome measures}

Sensitivity, specificity, and positive and negative predictive values.

\section{Main results}

38 patients (48\%) fulfilled NINCDS-ADRDA criteria for probable $\mathrm{AD}, 7(9 \%)$ fulfilled NINDS-AIREN criteria for probable $\mathrm{VaD}$, and $2(3 \%)$ fulfilled CDLB for probable DLB. 27 patients $(34 \%)$ had mixed pathology. The table shows sensitivity, specificity, and positive and negative predictive values of the tests.

\section{Conclusions}

Clinical diagnostic criteria for Alzheimer's disease, vascular dementia, and dementia with Lewy bodies had low sensitivity and relatively high specificity. Approximately one third of patients had mixed pathology.

Sources of funding:Medical Research Council (UK) and the Wellcome Trust.

For correspondence: Dr C Holmes, University of Southampton, Thornhill Research Unit, Moorgreen Hospital, Botley Road, West End, Southampton SO30 3JB, UK. Fax +44 (0)1703 475482.

Sensitivity, specificity, and positive and negative predictive values for clinical diagnosis compared with postmortem examination*

\begin{tabular}{|c|c|c|c|c|c|}
\hline Clinical diagnosis & Detected neuropathology & Sensitivity $(95 \%$ CI) & Specificity $(C I)$ & $P P V(C I)$ & $N P V(C I)$ \\
\hline \multirow[t]{2}{*}{ NINCDS probable } & Neuritic plaques alone & $66 \%(50$ to 80$)$ & $75 \%$ (58 to 88$)$ & $76 \%$ (59 to 89$)$ & $64 \%$ (48 to 78$)$ \\
\hline & Neuritic plaques alone and with other pathologies & $50 \%(37$ to 62$)$ & $70 \%(35$ to 93$)$ & $92 \%(78$ to 98$)$ & $17 \%(7$ to 31$)$ \\
\hline \multirow[t]{2}{*}{ AIREN probable } & Infarctions alone & $43 \%(10$ to 82$)$ & $95 \%(87$ to 98$)$ & $43 \%(10$ to 82$)$ & $95 \%$ (86 to 98$)$ \\
\hline & Infarctions alone and with other pathologies & $30 \%(13$ to 53$)$ & $100 \%(94$ to 100$)$ & $100 \%(59$ to 100$)$ & $78 \%(67$ to 87$)$ \\
\hline \multirow[t]{2}{*}{ DLB probable } & Lewy bodies alone and with neuritic plaques & $22 \%(3$ to 60$)$ & $100 \%(95$ to 100$)$ & $100 \%(16$ to 100$)$ & $91 \%(82$ to 96$)$ \\
\hline & Lewy bodies alone and with other pathologies & $16 \%(2$ to 48$)$ & $100 \%(95$ to 100$)$ & $100 \%(16$ to 100$)$ & $87 \%(77$ to 94$)$ \\
\hline
\end{tabular}

$* \mathrm{PPV}=$ positive predictive value; $\mathrm{NPV}=$ negative predictive value.

\section{Commentary}

This study by Holmes $e t$ al addresses the correspondence between clinical and pathological criteria for $\mathrm{AD}, \mathrm{VaD}$, and DLB. This work is reminiscent of a study by Litvan $e t$ al in which raters used data from 15 cases pathologically proved to have Parkinson's disease, 14 with DLB, and 76 with neither. ${ }^{1}$ For DLB, as in the present study, sensitivity was low and specificity was high. Mixed pathologies were considered in the context of parkinsonian syndromes, and inter-rater reliabilities were also considered. For AD, Nagy et al found high sensitivity (91-98\%), low specificity (40-61\%), and low negative predictive values for both the NINCDS-ADRDA and DSM-III-R criteria. ${ }^{2}$ The higher sensitivity found might have been from having less mixed pathology. For VaD, comparisons between NINDS-AIREN criteria and 3 other current criteria showed poor con- cordance, again perhaps reflecting the problem of mixed disease discussed by the present authors. ${ }^{3}$

Clinicians should appreciate the current work because it stresses the importance of (1) recognising disease prevalence, (2) appreciating the complexity introduced by mixed pathology, and (3) encouraging the clinician to consider the patient's values in tactfully informing the patient, or the patient's family, about the possibility of false positives and negatives when proceeding from a differential diagnosis to a provisional diagnosis. Although physicians should be cognisant of these and other diagnostic guidelines, this study does not support shifting the standard of care from the currently recommended one (general medical, neurological, and psychiatric assessment and an informed consent process indicating the nature of the differential diagnoses, relevant evaluation, and treatment alternatives and their prospective risks and benefits) to an algorithmic dependence on a specific set of guidelines. ${ }^{45}$

Steven Berman, MD

Louisiana State University Shreveport, Louisiana, USA

Harold J Bursztajn, MD

Harvard Medical School Cambridge, Massachusetts, USA

1 Litvan I, MacIntyre A, Goetz CG, et al. Arch Neurol 1998;55:969-78.

2 Nagy Z, Esiri MM, Hindley NJ, et al. Dement Geriatr Cogn Disord 1998:9:219-26.

3 Wetterling T, Kanitz RD, Borgis KJ. Stroke 1996; 27:30-6.

4 Practice parameter for diagnosis and evaluation of dementia (summary statement). Neurology 1994;44:2203-6.

5 Bursztajn HJ, Feinbloom RI, Hamm RM, et al. Medical choices, medical chances: how patients, families, and physicians can cope with uncertainty. families, and physicians can cope
New York: Routledge, 1990. 